\title{
Localisation aberrante de la connexine 43 dans le cancer du testicule.
}

\author{
C. ROGER1, B. MOGRABI1, D. CHEVALLIER1, J. MICHIELS2, D. SEGRETAIN1, \\ G. POINTIS1, P. FÉNICHEL1 \\ 1INSERM EMI 00-09, IFR 50, Faculté de Médecine, 2Laboratoire d'Anatomo-pathologie, \\ Hôpital de L'Archet, Nice
}

\section{RÉSUMÉ}

La communication intercellulaire via les jonctions gap (GJIC, pour «Gap Jonction Intercellular Communication ") est un mode ubiquitaire de dialogue entre les cellules. Il participe au contrôle de la prolifération cellulaire. Des troubles de l'expression des connexines $(\mathrm{Cx})$, les protéines transmembranaires constitutives de ces canaux intercellulaires, ont souvent été associés au phénotype malin. Dans le tube séminifère, la $\mathrm{Cx43}$ est exprimée majoritairement par les cellules de Sertoli et les cellules germinales basales. L'expression de la $\mathrm{Cx} 43$ a été étudiée dans une série de 4 cancers du testicule comprenant une prolifération séminomateuse exclusive, à un stade T1-2 N0 M0. Les transcrits et la protéine analysés par RT-PCR et Western blot, ont été retrouvés dans tous les cas, à la taille attendue. La protéine étudiée par immunofluorescence indirecte était délocalisée au sein du cytoplasme, non exprimée à la membrane, excluant sa participation à une éventuelle GJIC. La signification de cette localisation aberrante de la $\mathrm{Cx} 43$ est discutée dans le cadre des relations GJIC/tumorigenèse.

Mots clés : cancer testiculaire, séminome, communication jonctionnelle intercellulaire par gap, suppresseur tumoral, connexine 43.

\section{INTRODUCTION}

Dans la plupart des espèces et quelque soit l'organe, les cellules orientées vers la même fonction, sont capables de coordonner leur action, en échangeant des informations via des jonctions communicantes ou « jonctions gap ». Ces structures membranaires spécialisées, permettent le passage direct, de cytoplasme à cytoplasme, de molécules de signalisation de faible poids moléculaire $(<1 \mathrm{kDa})$, telles que le calcium, l'AMP cyclique, le GMP cyclique ou l'IP3, connues pour participer au contrôle du cycle cellulaire. La communication jonctionnelle intercellulaire par gap (GJIC, pour " Gap Jonction intercellular Communication ") joue ainsi un rôle de synchronisation particulièrement important au sein du myocarde, du myomètre, du système nerveux central ou des glandes endocrines. Elle intervient également dans le contrôle de la croissance et de la différentiation cellulaires. Des perturbations de cette communication ont souvent été retrouvées dans les processus de prolifération maligne $[9,34]$.

Correspondance : P. Fénichel, INSERM EMI 00-09, IFR 50, Faculté de Médecine, 28 avenue de Valombrose , 06107 Nice Cedex 2

Communication orale sélectionnées au XVIIème Congrès de la SALF, 7-8 décembre 2000, Bordeaux. 


\section{COMMUNICATION JONCTIONNELLE INTERCELLULAIRE PAR GAP ET CONNEXINES}

Les jonctions communicantes établissent de véritables canaux intercellulaires regroupés sous forme de plaques jonctionnelles membranaires. La jonction gap est formée par le couplage de deux hémi-canaux, ou connexons, situés de part et d'autre des deux membranes. Les connexons sont composés de six connexines $(\mathrm{Cx})$ identiques ou différentes formant ainsi un homo- ou hétéro-hexamère. Selon la composition du connexon en $\mathrm{Cx}$, la perméabilité du canal peut varier. Les $\mathrm{Cx}$ représentent une famille de plus de quinze protéines transmembranaires codées par des gènes différents [4] et caractérisées par leurs poids moléculaires ( $\mathrm{Cx} 43, \mathrm{Cx}$ de $43 \mathrm{kDa})$. Une $\mathrm{Cx}$ est composée de quatre domaines transmembranaires, deux boucles extracellulaires et une boucle intracellulaire et des domaines amino- et carboxy-terminaux intracytoplasmiques (Figure 1). L'extrémité C-terminale possède des sites de phosphorylation qui sont impliqués dans l'ouverture ou la fermeture rapide du canal par l'intermédiaire de kinases $[18,19]$.

Les $\mathrm{Cx}$ présentent une régulation tissu-spécifique. La Cx43 est exprimée ubiquitairement et est la représentante de cette famille, la plus étudiée à ce jour. Elle joue un rôle essentiel, en particulier, au niveau du myocarde comme le démontrent les troubles à la naissance des souris knock-out Cx43-/-, qui meurent de malformations cardiaques [27]. Elle est exprimée également au niveau du foie [3], du follicule ovarien [8], du myomètre où, sous l'effet des estrogènes, elle coordonne les contractions utérines. De plus, elle est majoritairement exprimée dans le testicule de rongeur $[28,1]$ et le testicule humain [32].

\section{COMMUNICATION JONCTIONNEL- LE INTERCELLULAIRE PAR GAP ET CANCER}

Des perturbations de la communication jonctionnelle ont souvent été observées au sein de cellules transformées ou cancéreuses, entre elles, ou avec les cellules saines avoisinantes $[9,21,5]$. Ces troubles de la GJIC peuvent être liés soit à une altération de l'expression du gène de certaines $\mathrm{Cx}$, soit à des modifications post-traductionnelles, comme des anomalies de l'état de phosphorylation [11, 29], ou des troubles du trafic des Cx qui ne sont alors plus localisées à la membrane mais délocalisées dans le cytoplasme [16]. Différents promoteurs tumoraux comme le TPA sont capables d'induire également ces anomalies [17, 26]. Les $\mathrm{Cx}$ sont aussi les cibles potentielles de différents oncogènes (src, ras, gay, neu) conduisant à la fermeture des canaux par hyperphosphorylation du domaine C-terminal [13, 14]. Par ailleurs, dans les différents modèles cellulaires malins où une anomalie de la GJIC a été mise en évidence, la réexpression voire la surexpression de certaines $\mathrm{Cx}$ par transfert de gènes, conduit soit à un ralentissement soit à une normalisation de la prolifération cellulaire in vitro [30] et in vivo [9]. Ces observations suggèrent que les $\mathrm{Cx}$ peuvent être considérées comme de véritables suppresseurs tumoraux [22].

\section{LA SPERMATOGENÈSE, UN PROCESSUS CONTRÔLÉ}

La spermatogenèse est un processus cyclique de prolifération et de différenciation cellulaires étroitement contrôlé sur le plan spatio-temporel. Ce contrôle est à la fois de nature endocrine via la FSH et paracrine via la testostérone, les facteurs de croissance et les cytokines présents au sein du tube séminifêre. À l'âge adulte chez le sujet normal, la prolifération des cellules germinales basales pré-méiotiques est stimulée de façon intermittente dans un tube donné et à un niveau donné du tube, pour permettre le démarrage d'une nouvelle vague de spermatogenèse. Des études ultrastructurales avaient montré depuis longtemps la présence de jonctions gap au sein de l'épithélium séminifêre [23]. Récemment, l'approche moléculaire des Cx a permis de suggérer leur participation au contrôle sertolien de la spermatogenèse $[2$, 28]. Un phénomène similaire a été mis en évidence au niveau du follicule ovarien, où la GJIC participe au blocage et à la reprise de la 


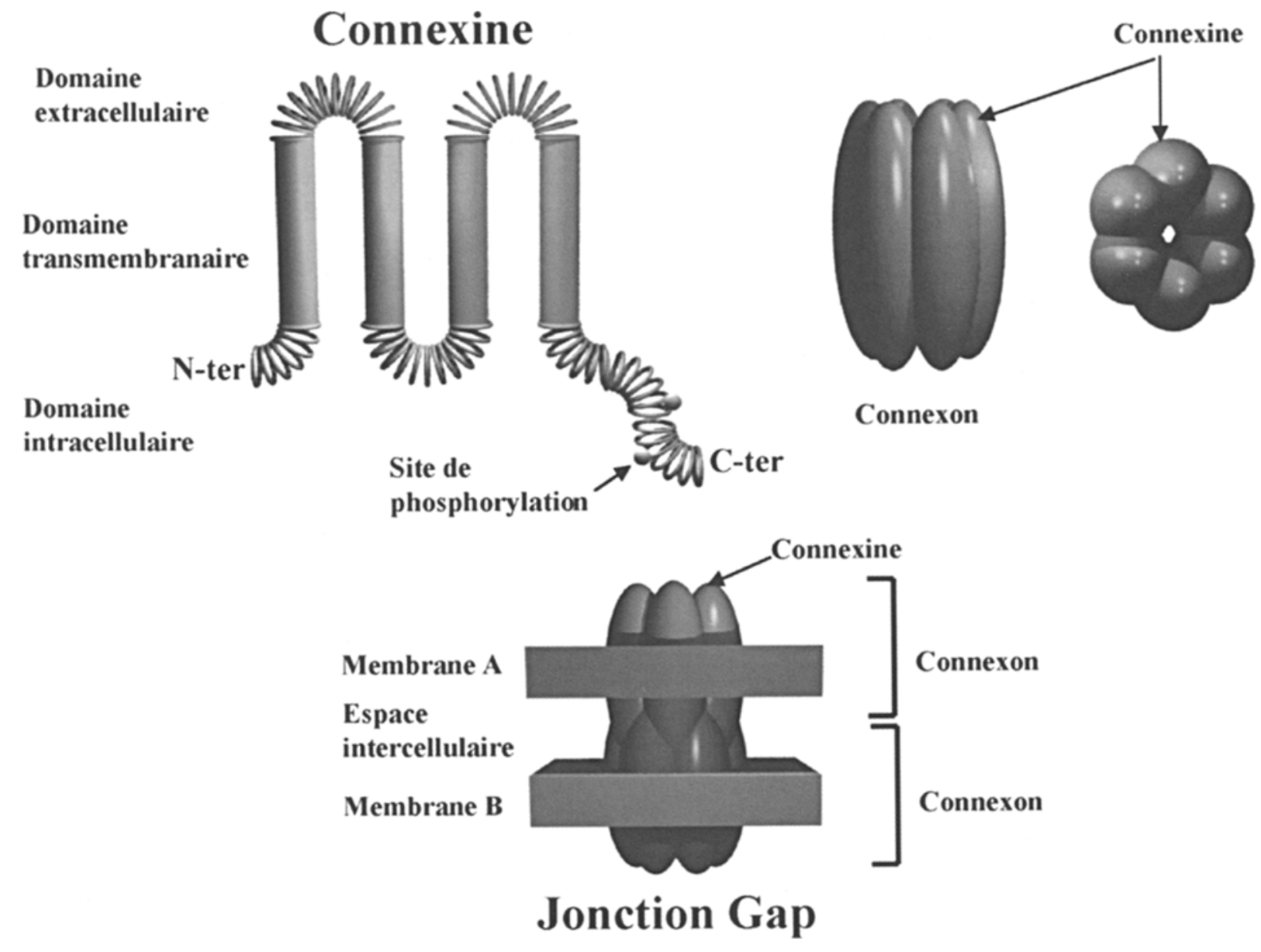

Figure 1 : Représentation des connexines et de la jonction Gap.

méiose, par l'intermédiaire des $\mathrm{Cx} 43$ et 37 exprimées au niveau des cellules de la granulosa équivalentes des cellules de Sertoli, et de l'ovocyte [30, 22]. De plus, l'observation des tubes séminifères de souris transgéniques homozygotes mâles, pour lesquelles la région codante de la $\mathrm{Cx} 43$ a été remplacée respectivement par les régions codantes de la $\mathrm{Cx} 32$ et la $\mathrm{Cx} 40$, a permis de constater une altération de la spermatogenèse identique au syndrome des cellules de Sertoli isolées [25]. L'ensemble de ces données tendent à démontrer le rôle primordial de la $\mathrm{Cx} 43$ dans les processus de développement des cellules germinales, aussi bien chez le mâle que chez la femelle.

À ce jour, cinq $\mathrm{Cx}$ ont été caractérisées dans le testicule de rongeur ( $\mathrm{Cx} 26,32,33,37$ et 43$)$. La Cx43 est la plus représentée ; elle est présente à la fois dans le compartiment interstitiel au niveau des cellules de Leydig et dans le tube séminifêre au niveau des cellules de Sertoli et des cellules germinales basales, sper- matogonies et spermatocytes de premier ordre [2]. Elle est exprimée à l'identique dans le testicule humain normal [Defamie et coll., soumis pour publication]. Son expression varie au cours des stades de la spermatogenèse [2] et des anomalies ont été retrouvées en cas d'altérations de la spermatogenèse $[1,2]$.

\section{EXPRESSION DE LA CX43 DANS LE SEMINOME HUMAIN}

Le cancer du testicule représente le cancer le plus fréquent de l'adulte jeune. En Europe, son incidence augmente de $2 \%$ par an (rapport du ministère danois de l'environnement et de l'énergie, Miljoprojekt 1995, NR290). 90\% des cancers du testicule sont des séminomes dont on distingue la forme pure (50\%). Il s'agit alors d'une prolifération anarchique des seules cellules germinales indifférenciées.

La caractérisation de la $\mathrm{Cx} 43$ au niveau Sertolien et germinal et sa possible implication dans le contrôle de la spermatogenèse, 
nous a amené à émettre l'hypothèse qu'une perte partielle du contrôle sertolien de la spermatogenèse via une altération de la GJIC établie entre Sertoli/germinale, pourrait être à l'origine, participer ou être associée au processus de prolifération séminomateuse.

Nous avons donc étudié l'état de la GJIC et plus particulièrement l'expression de la $\mathrm{Cx} 43$ dans 4 séminomes purs prélevés chez des adultes jeunes ne présentant pas d'invasion métastatique. À partir de tissus séminomateux, congelés à l'état frais, nous avons confirmé par RT-PCR, dans tous les cas, la présence de transcrits de la $\mathrm{Cx} 43$ humaine, à la taille attendue de 1157 pb (Figure 2). L'analyse par Western-Blot, a révélé la présence de la protéine à un poids moléculaire de $43 \mathrm{kDa}$ et de ses différentes formes phosphorylées (Figure 3). Par contre, par immunofluorescence indirecte, la protéine a été localisée dans le cytoplasme et aucun signal n'a été retrouvé au niveau membranaire (Figure 4). Afin de préciser le trouble du trafic intracellulaire impliqué dans cette délocalisation (exocytose, endocytose, dégradation), la co-localisation de la $\mathrm{Cx} 43$ avec des marqueurs des différents compartiments intracytoplasmiques, est actuellement en cours.

La Cx43 est donc exprimée dans le séminome aussi bien en terme de transcrits que de protéine, mais sa localisation est intracytoplasmique et non membranaire, excluant donc sa participation à une GJIC fonctionnelle. Cette observation soulève au moins deux questions : quels sont les mécanismes de cette délocalisation et est-elle la conséquence de l'état transformé de la cellule ? le trouble de l'adressage de la protéine à la membrane participe-t-il au processus néoplasique?

Le tableau 1 rapporte les cancers pour lesquels une anomalie d'expression de la $\mathrm{Cx} 43$ a été mise en évidence. Dans certains modèles, on observe une inhibition partielle de la transcription, des modifications de l'état de phos- phorylation de la protéine ou une délocalisation de celle-ci. Une localisation aberrante a également été caractérisée pour d'autres $\mathrm{Cx}$ dans des cellules tumorales [12].

Il est possible d'envisager une mutation somatique portant sur une partie du gène codant pour un domaine impliqué dans l'adressage de la protéine à la membrane comme rapporté par Krutovskikh [16]. Cependant, l'association Cancer/mutation des Cx de semble rare.

Une autre hypothèse concernant cette délocalisation cytoplasmique pourrait être la présence d'anomalies portant sur les protéines associées au transport membranaire. Il est possible que certaines protéines, décrites pour être associées ou participant au transport membranaire des $\mathrm{Cx}$ (Zonula Occludens 1, N-cadherine) [33;7], soient ici non exprimées ou anormales. De plus, les différents états de phosphorylation des $\mathrm{Cx}$ observés dans les processus malins, pourraient être à l'origine d'une modification de leur conformation empêchant ainsi toute liaison avec ces protéines associées.

Quant à la participation de la Cx43 en tant que facteur suppresseur tumoral, différents travaux sur des modèles malins ont montré l'effet du rétablissement de l'expression de la $\mathrm{Cx} 43$ et de la GJIC, sur la réduction de la prolifération cellulaire [35]. Le rétablissement de la GJIC, via la surexpression de la $\mathrm{Cx} 43$, jouerait un rôle direct sur le contrôle des différentes phases du cycle cellulaire en modulant l'expression des cyclines A, D1 et D2 [15, 6].

En conclusion, différentes données montrent que de nombreux promoteurs tumoraux et/ou pesticides sont capables d'induire des altérations de la GJIC. Leur rôle dans la promotion des cancers testiculaires a été suggéré. Nous rechercherons les causes de la localisation aberrante de la $\mathrm{Cx} 43$ dans le séminome humain et tenterons d'établir une relation entre elle et la prolifération séminomateuse maligne. 
Figure 2 : Analyse de l'expression de la Cx 43 par RT-PCR dans le testicule humain et dans 4 séminomes humains. Ctrl : testicule humain (1157 pb) ; S1-S4 : séminome congelé à l'état frais.

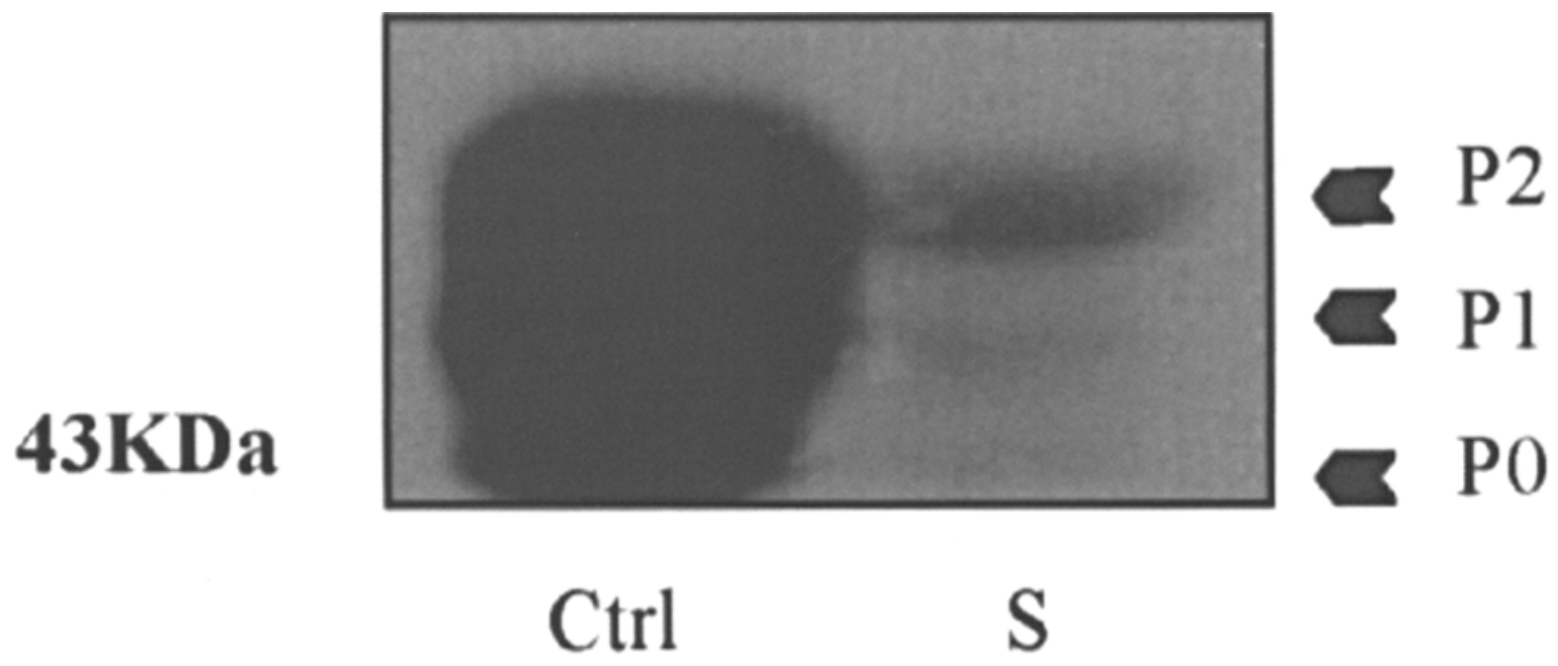

Figure 3 : Analyse de la Cx 43 par Western Blot dans le testicule humain et dans le séminome. Dépôt de 150 $\mu$ g de protéines. Ctrl : Testicule humain ; S : Séminome. P0 : forme non-phosphorylée de la Cx 43. P1-P2 : formes phosphorylées de la Cx 43.
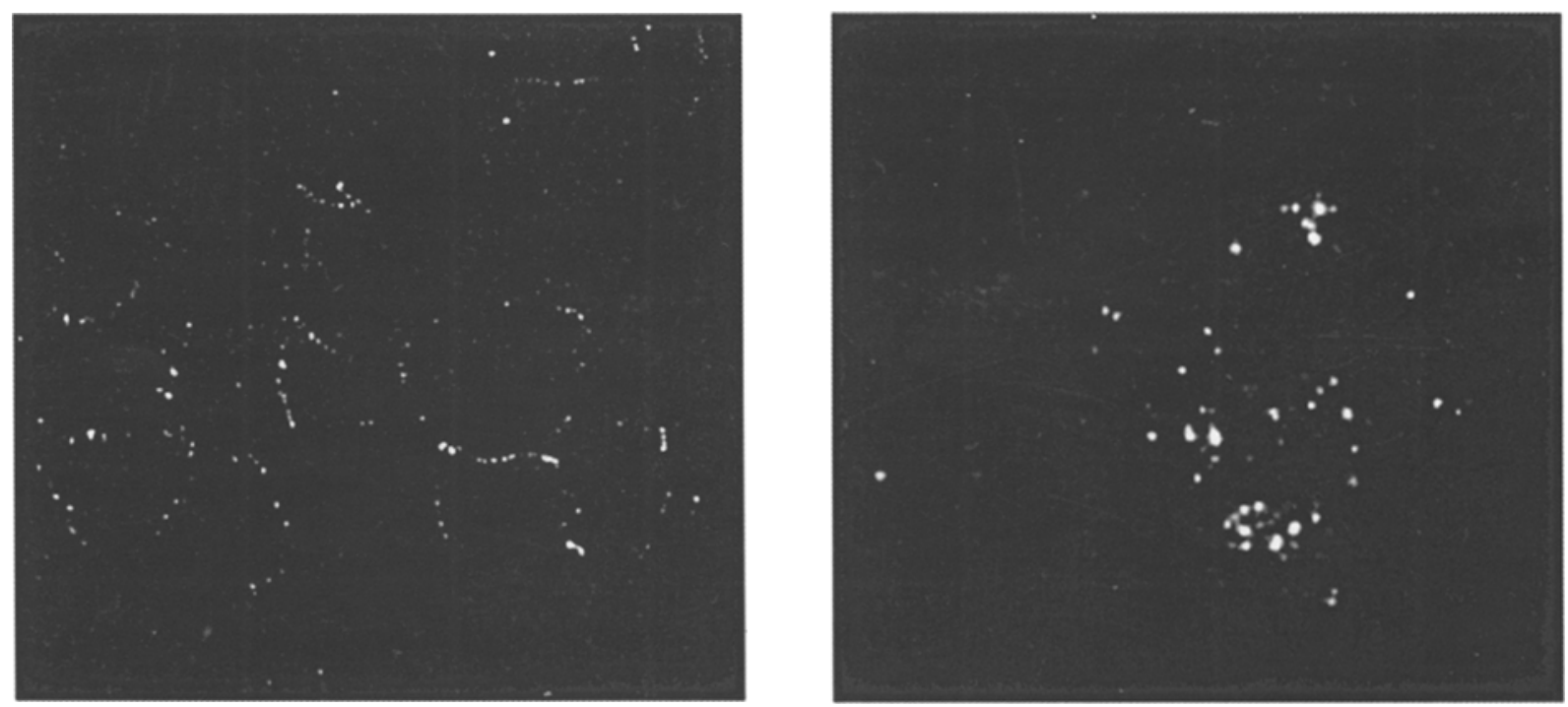

Figure 4 : Localisation de la Cx 43 en immunofluorescence. 1 : Cellules contrôle, notez le marquage membranaire de la Cx 43 caractéristique des jonctions Gap. 2 : Cellule séminomateuse, la Cx 43 est localisée dans le cytoplasme. 


\begin{tabular}{|c|c|c|c|c|c|c|}
\hline organe & tissu ou lianée & ARN & Protéine & localisation & fonctionalité & Référence \\
\hline cerveau & C6 glioma cell & $\begin{array}{c}\text { fable niveau d'ARNm } \\
\text { de la } \mathrm{C} \times 43\end{array}$ & présente & membrane & faible & $\begin{array}{c}\text { D.Zhu et coll } \\
\text { Proc. Nat Acad Sci. USA } \\
\text { vol.88 pp.1883-1887 } \\
\text { march } 1991\end{array}$ \\
\hline sein & $\begin{array}{l}\text { normal } \\
\text { carcinoma }\end{array}$ & $\begin{array}{c}\text { exprime } \\
\text { non exprime }\end{array}$ & $\begin{array}{c}\text { presente } \\
\text { non presente }\end{array}$ & $\begin{array}{c}\text { membrane } \\
- \\
\end{array}$ & fonctionnelle & $\begin{array}{c}\text { Sw.Lee of coll } \\
\text { J Cell Biol } \\
1992 \text { Sep;118(5):1213-21 }\end{array}$ \\
\hline prostate & $\begin{array}{l}\text { normal } \\
\text { PC3 } \\
\text { LNCaP } \\
\text { TSU-RI } \\
\text { ALVA-31 }\end{array}$ & $\begin{array}{l}\text { present } \\
\text { nd } \\
\text { nd } \\
\text { present } \\
\text { present }\end{array}$ & $\begin{array}{c}3 \text { formes phosphorylees } \\
\text { non presente } \\
\text { non presente } \\
\text { forme non phosphorylee } \\
\text { forme non phosphorvlee }\end{array}$ & $\begin{array}{l}\text { membrane } \\
\text { nd } \\
\text { nd } \\
\text { nd } \\
\text { nd }\end{array}$ & $\begin{array}{l}\text { fonctionnelle } \\
\text { nd } \\
\text { non fonctionnelle } \\
\text { nd } \\
\text { nd }\end{array}$ & $\begin{array}{l}\text { M.Z. Hossain et coll } \\
\text { the prostate } \\
38.55-59 \text { (1999) }\end{array}$ \\
\hline $\begin{array}{l}\text { endometre } \\
\text { humain }\end{array}$ & $\begin{array}{l}\text { normal } \\
\text { FEEC } \\
\text { HEC-1A } \\
\text { RL-95-2. }\end{array}$ & $\begin{array}{c}\text { present } \\
\text { present } \\
-\end{array}$ & $\begin{array}{c}3 \text { formes phosphorylees } \\
\text { forme non phosphorylee } \\
\text { non présente } \\
\text { non présente }\end{array}$ & $\begin{array}{c}\text { membrane } \\
\text { membrane } \\
.\end{array}$ & $\begin{array}{l}\text { nd } \\
\text { nd } \\
\text { nd } \\
\text { nd }\end{array}$ & $\begin{array}{l}\text { SR. Schlemmer et coll } \\
\text { Exp Mol Pathol } \\
1999 \text { Dec:67(3):150-63 }\end{array}$ \\
\hline ovaire humain & $\begin{array}{c}\text { HOSE (normal) } \\
\text { CaOV-3 } \\
\text { OVCAR-3 } \\
\text { SK-OV-3 } \\
\text { SW626 }\end{array}$ & $\begin{array}{c}\text { forte expression } \\
- \\
- \\
- \\
-\end{array}$ & $\begin{array}{l}\text { nd } \\
\text { nd } \\
\text { nd } \\
\text { nd } \\
\text { nd }\end{array}$ & $\begin{array}{l}\text { nd } \\
\text { nd } \\
\text { nd } \\
\text { nd } \\
\text { nd }\end{array}$ & $\begin{array}{c}\text { fonctionnelle } \\
- \\
- \\
- \\
-\end{array}$ & $\begin{array}{c}\text { E. A Hanna ef coll } \\
\text { Carcinogenesis } \\
1999 \text { Jul;20(7):1369-73 }\end{array}$ \\
\hline rein humain & $\begin{array}{l}\text { ACHN } \\
\text { NT }\end{array}$ & $\begin{array}{c}\text { normal } \\
\text { non detectable }\end{array}$ & $\begin{array}{c}\text { non phosphorylee } \\
\text { non detectable }\end{array}$ & cytoplsamique & - & $\begin{array}{c}\text { MNoguchi el coll } \\
\text { Urology } \\
1999 \operatorname{Jan} 53(1): 218-22\end{array}$ \\
\hline $\begin{array}{l}\text { cellule } \\
\text { germinale }\end{array}$ & $\begin{array}{l}\text { normal } \\
\text { seminome }\end{array}$ & $\begin{array}{c}\text { nd } \\
\text { present }\end{array}$ & $\begin{array}{c}\text { nd } \\
\text { presente }\end{array}$ & $\begin{array}{c}\text { nd } \\
\text { cytoplasmique }\end{array}$ & $\begin{array}{c}\text { nd } \\
\text { non fonctionnelle }\end{array}$ & \\
\hline
\end{tabular}

Tableau 1 : Anomalies de la Cx 43 dans les cancers. Les données concernant les cellules germinales et séminomateuses sont présentées dans ce travail (INSERM EMI 00-09).

\section{RÉFÉRENCES}

1. BATIAS C., DEFAMIE N., LABLACK A., et al. : Modified expression of testicular gap junction connexin 43 during normal spermatogenic cycle and in altered spermatogenesis. Cell. Tissue Res., 1999, 298:113-121.

2. BATIAS C., SIFFROI J.P., FÉNICHEL P., POINTIS G., SEGRETAIN D. : Connexin 43 gene expression and regulation in the roddent seminiferous epithelium. J. Histochem. Cytochem., 2000, 48:793-805.

3. BERTHOUD V.M., LEDBETTER M.L., HERTZBERG E.L., SAEZ J.C. : Connexin 43 in MDCK cells: regulation by a tumor-promoting phorbol ester and $\mathrm{Ca} 2+$. Eur. J. Cell. Biol., 1992, $57: 40-50$.

4. BRUZZONE R., WHITE TW, PAUL D. : Connexions with connexins: the molecular basis of direct intercellular signaling. Eur. J. Biochem., 1996, 238:1-27.

5. COCHAND-PRIOLLET B., RAISON D., MOLINIE V., GUILLAUSSEAU P.J., WASSEF M., BOUCHAUD C. : Altered gap and tight junctions in human thyroid oncocytic tumors: a study of 8 cases by freeze-fracture. Ultrastruct. Pathol., 1998, 22:413-20.

6. CHEN S.C., PELLETIER D.B., AO P., BOYNTON A.L. : Connexin 43 reverses the phenotype of transformed cells and alters cyclin-dependent kinases. Cell Growth Differ. 1995, 6:681-690.

7. GIEPMANS B.N., MOOLENAAR W.H. : The gap junction protein connexin 43 interacts with the second PDZ domain of the zona occludens-1 protein. Curr. Biol., 1998, 8:931-4

8. GRANOT I., DEKEL N. : Developmental expression and regulation of the gap junction protein and trans- cript in rat ovaries. Mol. Reprod. Dev., 1997, 47:231-9.

9. HOTZ-WAGENBLATT A., SHALLOWAY D. : Gap junctional communication and neoplastic transformation. Critical Reviews in Oncogenesis, 1993, 5:541-558.

10. HANNA E.A., UMHAUER S., ROSHONG S.L., et al. : Gap junctional intercellular communication and connexin 43 expression in human ovarian surface epithelial cells and ovarian carcinomas in vivo and in vitro. Carcinogenesis, 1999, 20:1369-73.

11. HOSSAIN M.Z., JAGDALE A., PENG A.O, LECIEL C., RUO-PAN HUANG, BOYNTON A.L. : Impaired expression and postranslational processing of Connexin43 and downregulation of gap junctional communication in neoplastic human prostate cells. Prostate, 1999, 38:55-59

12. JAMIESON S., GOING J.J., D'ARCY R., GEORGE W.D. : Expression of gap junction proteins connexin 26 and connexin 43 in normal human breast and in breast tumours. J. Pathol., 1998, 184:37-43.

13. JOU Y.S., LAYHE B., MATESIC D.F, et al. : Inhibition of gap junctional intercellular communication and malignant transformation of rat liver epithelial cells by neu oncogene. Carcinogenesis, 1995,16:311-317.

14. KANEMITSU M.Y., LOO L.W., SIMON S., LAU A.F., ECKHART W. : Tyrosine phosphorylation of connexin 43 by v-Src is mediated by SH2 and SH3 domain interactions. J. Biol. Chem., 1997, 272:22824-22831

15. KOFFLER L., ROSHONG S., KYU PARK I., et al. : Growth inhibition in $\mathrm{G}(1)$ and altered expression of cyclin D1 and p27(kip-1) after forced connexin expression in lung and liver carcinoma cells. J. Cell. Biochem., 2000, 79:347-354

16. KRUTOVSKIKH V.A., TROYANOVKI S.M., PICCOLI 
C., et al. : Differential effect of subcellular localization of communication impairing gap junction protein connexin 43 on tumor cell growth in vivo. Oncogene. 2000, 19:505-513.

17. LABLACK A., BOURDON V., DEFAMIE N., et al. : Ultrastructural and biochemical evidence for gap junction and connexin 43 expression in a clonal Sertoli cell line: a potential model in the study of junctional complex formation. Cell. Tissue Res. 1998, 294:279-287.

18. LAMPE P.D., TENBROEK E.M., BURT J.M., KURATA W.E., JOHNSON R.G., LAU A.F. : Phosphorylation of connexin 43 on serine 368 by protein kinase $\mathrm{C}$ regulates gap junctional communication. J. Cell. Biol., 2000, 149:1503-1512.

19. LAU A., KURATA W.E., KANEMITSU M.Y., et al. : Regulation of connexin 43 function by activated tyroine protein kinases. J. Bioenerg. Biomembr., 1999, 28:359-368.

20. LEE S.M., TOMASETTO C., SAGER R. : Positive selection of candidate tumor suppressor genes by subsractive hybridization. Proc. Natl. Acad. Sci. USA, 1991, 88:2825-2829.

21. LEE S.W., TOMASETTO C., PAUL D., KEYOMARSI K., SAGER R. : Transcriptional downregulation of gapjunction proteins blocks junctional communication in human mammary tumor cell lines. J. Cell. Biol., 1992, 118:1213-1221.

22. LI R., MATHER J.P. : Lindane, an inhibitor of gap junction formation, abolishes oocyte directed follicle organizing activity in vitro. Endocrinology, 1997, 138:4477-4480

23. McGINLEY D., POSALAKY Z., PORVAZNIK M, RUSSELL L. : Gap junctions between Sertoli cell and germ cells of rat seminiferous tubules. Tissue Cell., 1977, 11:741-754.

24. NOGUCHI M., NOMATA K., WATANABE J.I., SATO H., KANETAKE H., SAITO Y. : Disruption of gap junctional intercellular communication in human renal cancer cell lines. Urology, 1999, 53:218-222.

25. PLUM A., HALLAS G., MAGIN T., et al. : Unique and shared functions of different connexins in mice. Curr. Biol., 2000, 10:1083-1091.

26. REN P., MEHTA P.P., RUCH R.J. : Inhibition of gap junctional intercellular communication by tumor promoters in connexin 43 and connexin 32-expressing liver cells: cell specificity and role of protein kinase C. Carcinogenesis, 1998, 19:169-175.

27. REAUME A.G., DE SOUSA P.A., KULKARNI S et al. : Cardiac malformation in neonatal mice lacking connexin 43. Science 1995, 267:1831-1834.

28. RISLEY M.S., TAN I.P., ROY C., SAEZ J.C. : Cell-, age- and stage-dependent distribution of connexin 43 gap junctions in testes. J. Cell. Sci., 1992, 103:81-96

29. SCHLEMMER S.R., NOVOTNY D.B., KAUFMAN D.G. : Changes in connexin 43 protein expression in human endometrial carcinoma. Exp. Mol. Pathol., 1999, 67:150-63.

30. SIMON A.M., GOODENOUGH D.A., LI E., PAUL D.L. : Female infertility in mice lacking connexin 37. Nature, 1997, 385:525-529
31. STATUTO M., AUDEBET C.H., TON OLI H., SELMIRUBY S., ROUSSET B., MUNARI-SILEM Y. : Restoration of cell-to-cell communication in thyroid cell lines by tranfectionn with and stable expression of the connexine 32. J. Cell. Biol., 1997, 272, 2471024716.

32. STEGER K., TETENS F., BERGMANN M. : Expression of connexin 43 in human testis. Histochem. Cell. Biol., 1999, 112:215-220.

33. TOYOFUKU T., YABUKI M., OTSU K., KUZUYA T., HORI M., TADA M. : Direct association of the gap junction protein connexin-43 with ZO-1 in cardiac myocytes. J. Biol. Chem. 1998, 273 :12725-12731.

34. YAMASAKI H., KRUTOVSKIKH V., MESNIL M., TANAKA T., ZAIDAN-DABGLI M.L., OMORI Y. : Role of connexins (gap junction) genes in cell growth control and carcinogenesis. C.R. Acad. Sci. Paris, 1999, 322:1651-1659.

35. ZHU D., CAVENEY S., KIDDER G.M., NAUS C.G.C. : Transfection of $\mathrm{C} 6$ glioma cells with Connexin 43 cDNA: Analysis of expression, intercellular coupling, and cell proliferation. Proc. Natl. Acad. Sci USA, 1991, 88:1883-1887.

\section{ABSTRACT}

\section{Aberrant localization of connexin 43 in testi- cular cancer}

\begin{abstract}
ROGER C., MOGRABI B, CHEVALLIER D, MICHIELS JF, SEGRETAIN D, POINTIS G, FENICHEL $P$
\end{abstract}

Most cells can communicate directly via gap junction channels. Gap junction intercellular communication (GJIC) participates in the control of cell proliferation. Abnormal expression of connexins $(\mathrm{Cx})$, the constitutive proteins of gap junctions, has been associated with a transformed phenotype. In the seminiferous tubules, connexin $\mathrm{Cx} 43$ is predominantly expressed by Sertoli cell and germinal cell membranes. We studied $\mathrm{Cx} 43$ expression in four testicular cancers (pure seminoma). Cx43 mRNA and protein characterized by RT PCR and Western blot were found to be similar to controls (normal testes) in each case. However, immunofluorescence study of $\mathrm{Cx} 43$ protein indicated a cytoplasmic localization with no membrane expression, excluding the participation of $\mathrm{Cx43}$ in GJIC. The significance of this aberrant localization will be discussed in relation to carcinogenesis.

Key-words: Testicular cancer, seminoma, Gap junction intercellular communication, Tumor suppressor, Connexin 43 